\title{
Three-Dimensional Surface Imaging - An Objective Approach of Quality Assurance in Facial Plastic, Reconstructive and Aesthetic Surgery?
}

\author{
Laszlo KOVACS, Fee ARMBRECHT, Stefan RAITH, Alexander VOLF, Nikolaos A. PAPADOPULOS, \\ and Maximilian EDER \\ Research Group Computer Aided Plastic Surgery - CAPS, \\ Clinic for Plastic Surgery and Hand Surgery, Technical University Munich, Germany
}

\begin{abstract}
Background: Assessment of the aesthetic result of plastic, reconstructive and aesthetic surgery has been predominantly based on physician experience and judgment and shaped purely subjectively by the proficiency of the surgeon and the perception and satisfaction after surgery of the patient. Outcomes research is a fast-growing field of study that focuses on patient-related aspects of medical or surgical outcomes such as satisfaction and quality of life. Currently, standardized questionnaires try to objectively assess the quality outcome of surgery by collecting the subjective surgeon and patient estimation of the surgical outcome. The lack of a means to guarantee objective and exact recording as well as quantification of surgical modification hardly permits the evaluation of postoperative process controls, judgement of the order of precedence of competitive surgical methods and even comparing the results of different surgeons when measuring the surgical outcome by subjective endpoints. Three-Dimensional (3-D) surface imaging offers the ability to objectively capture anatomical structures and to evaluate changes regarding shape, surface, volume and symmetry after surgery. The goal of this study is to present the potential application of 3-D surface imaging as an objective evaluation tool for quality assurance and outcome research purposes in plastic, reconstructive and aesthetic surgery.
\end{abstract}

Materials and Methods: By means of clinical examples in different fields of plastic, reconstructive and aesthetic surgery we analysed the possibilities of 3-D surface imaging to support an objective pre- and postoperative clinical documentation, surgical planning tool and quality assurance method. The 3-D assessment of the region of interest was carried out using a 3-D linear laser scanner, Type Minolta Vivid $910^{\circledR}$ (Minolta Co., Ltd, Osaka, Japan). With the help of special software (Raindrop Geomagic Qualify7®, Raindrop Geomagic, Inc., NC, U.S.A) the geometrical attributes of the 3-D surface region of interest are generated and all quantifiable surgical changes (volume, surface, shape and the symmetry) are automatically summarized in a standardized and structured report (Fig. 1).

Results: The accurate and precise quantification and comprehensible visualization using the standardized report of the region of interest and surgical changes leads to a better interpretation of anatomical structures, patient specific preoperative planning and individualised treatment, clearly structured patient documentation (databank), interactive physician-patient relationship, objective postoperative process controls (swelling, edema, shape changes over time etc.) and evaluation of postoperative results in terms of an objective quality assessment of the surgical outcome (Fig. 2).

Conclusion: In facial plastic and reconstructive surgery, particularly aesthetic facial plastic surgery, patient satisfaction remains the benchmark by which a successful surgical outcome is measured. It has been suggested that patient-based outcome measurement (use of questionnaires, interview schedules or any associated way of quantifying health-related variables from the patient's perspective), may be the only appropriate method of evaluation because quality of life must be the endpoint. 3-D surface imaging provides the surgeon for the first time with additional objective quantification of the surgical result. This new approach delivers fundamental scientific data, combined with questionnaires to evaluate the subjective estimations of surgeons and patients, to enable an evidence based medical outcome research in plastic, reconstructive and aesthetic surgery.

Keywords: 3-D surface imaging, quality assurance, facial surgery, face, plastic surgery 


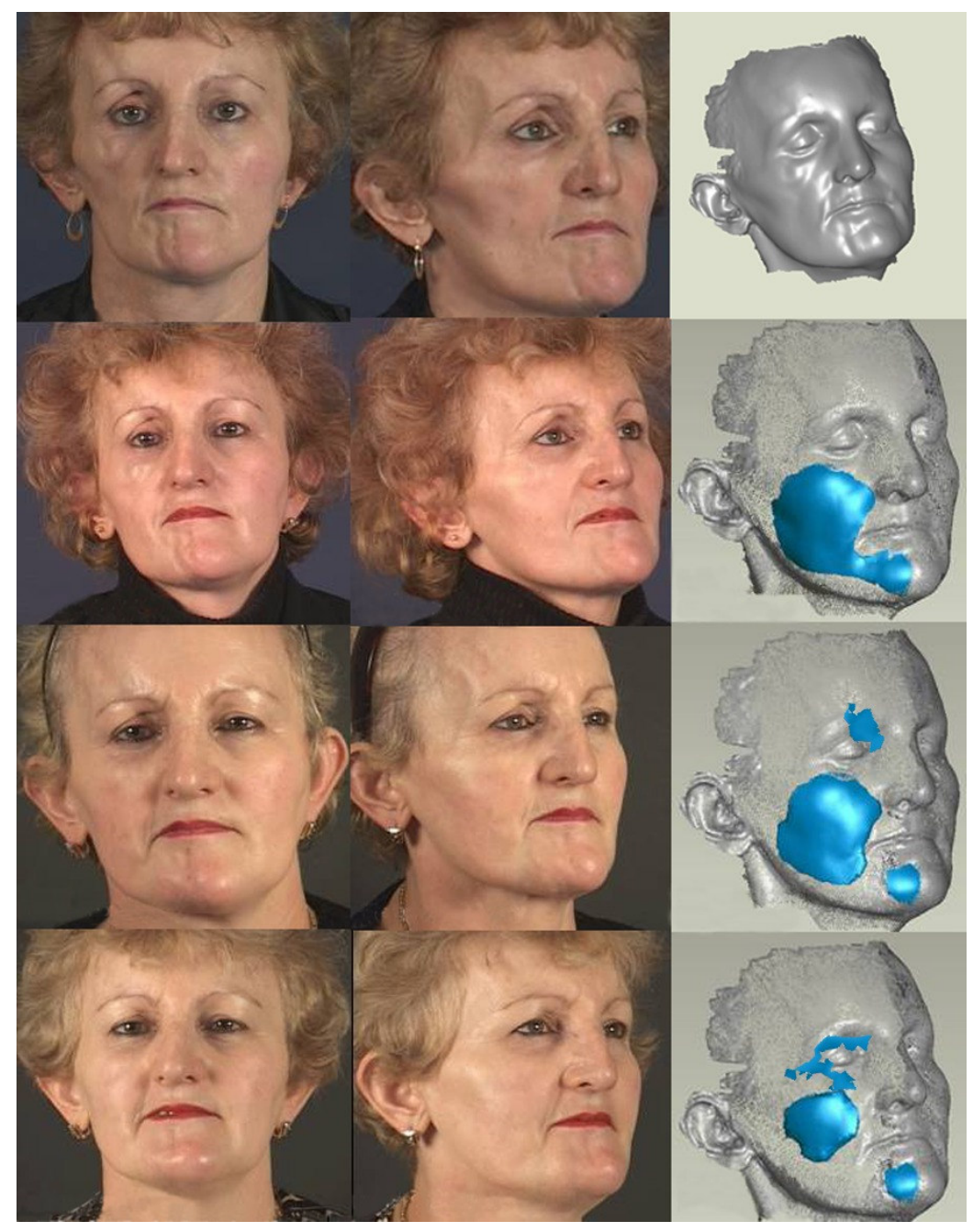

Fig. 1: Objective quantification of surgical changes

Quantification of soft-tissue defect and fat transplantation in cc

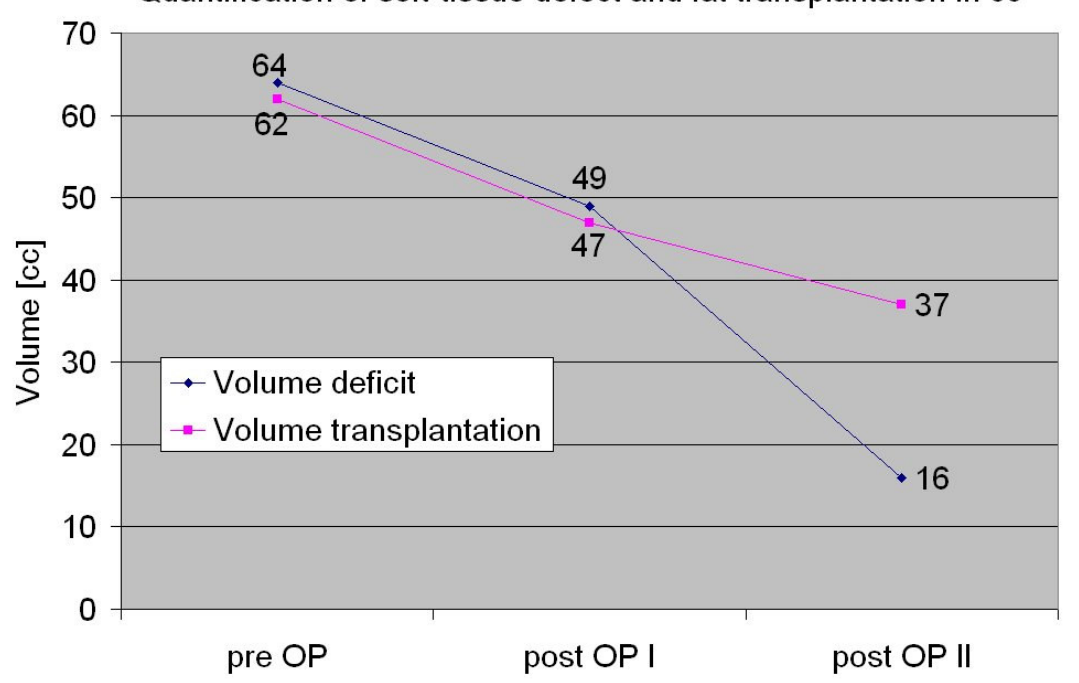

Fig. 2 Quantification of soft tissue changes after surgery 